\title{
Desfechos Adversos Perinatais Associados A Escolaridade Materna
}

\section{Adverse Perinatal Outcomes Associated With Maternal Education}

DOI: $10.54019 / \operatorname{sesv3n1-004}$

Recebimento dos originais: 05/12/2021

Aceitação para publicação: 20/02/2022

\section{Bianca Elicker Rosin}

Acadêmica de Medicina da Unversidade da Região de Joinville (UNIVILLE- Santa Catarina)

\section{André Luís Marcelo Albuquerque}

Acadêmico de Medicina da Unversidade da Região de Joinville (UNIVILLE- Santa Catarina)

\section{Rodrigo Ribeiro e Silva}

Acadêmico de Medicina da Unversidade da Região de Joinville (UNIVILLE- Santa Catarina)

\section{João Pedro Ribeiro Baptista}

Acadêmico de Medicina da Unversidade da Região de Joinville (UNIVILLE- Santa Catarina) - https://orcid.org/0000-0003-1760-4460

\section{Carla Gisele Vaichulonis}

Enfermeira graduada pela USP, Mestre em Saúde e Meio Ambiente pela Universidade da Região de Joinville (UNIVILLE- Santa Catarina) -

\section{Jean Carl Silva}

Médico graduado pela UFSC, Mestre em Saúde e Meio Ambiente pela Universidade da Região de Joinville (UNIVILLE- Santa Catarina), Doutor em Ciências Médicas pela UNIFESP, Professor de Obstetrícia do Curso de Medicina e Professor do curso de Pós-graduação em Saúde e Meio Ambiente da UNIVILLE

\section{RESUMO}

Objetivo: Comparar os diferentes níveis de escolaridade com os desfechos adversos perinatais em pacientes de baixo risco. Metodologia: Estudo de corte transversal, foram selecionadas aleatoriamente puérperas, de risco habitual, atendidas na rede pública, com gestação única. As puérperas avaliadas foram divididas em 3 grupos, de acordo com a escolaridade: primário ou inferior, secundário e superior ou mais. Os desfechos avaliados foram realização de cesariana, UTI neonatal, Apgar baixo de 1ำ minuto, prematuridade e baixo peso ao nascer. O cálculo da razão de chance teve intervalo de confiança de $95 \%$. Resultado: a população foi dividida em 3 grupos, 187 (52,9\%) puérperas com ensino primário ou menos, $437(60,5 \%)$ pacientes com ensino secundário e 98 $(13,6 \%)$ pacientes com ensino superior ou mais. As características maternas diferiram quanto a idade, gestações anteriores, partos normais, cesarianas 
prévias, idade $1^{\underline{a}}$ gestação, número de consultas de pré-natal, remuneração, número de pessoas morando na residência e uso de tabaco. No perfil do recémnascido, houve diferença somente quanto a cesariana. Após o cálculo de razão de chance, viu-se que pacientes com ensino primário ou inferior tiveram menor propensão a realização de cesariana (0,511 IC95\% 0,290-0,900), assim como as pacientes com ensino secundário (0,530 IC95\% 0,322-0,873), ambos comparados a população com ensino superior ou maior. Nos demais, desfechos não se observou significância. Conclusão: O ensino primário e o secundário mostraramse como fatores protetores para cesariana. Não encontramos diferença quanto a prematuridade, o baixo peso ao nascer, a necessidade de UTI neonatal e o Apgar baixo de $1^{\circ}$ minuto.

Palavras-chaves: puérperas, escolaridade, Metodologia

\section{ABSTRACT}

Objective: To compare different levels of education with adverse perinatal outcomes in low-risk patients. Methodology: Cross-sectional study, puerperae were randomly selected, usual risk, attended in the public network, with single gestation. The evaluated puerperae were divided into 3 groups, according to education: primary or less, secondary and higher or more. The outcomes evaluated were cesarean section, neonatal ICU, low 1st minute Apgar, prematurity, and low birth weight. The calculation of the odds ratio had a $95 \%$ confidence interval. Results: the population was divided into 3 groups, 187 (52.9\%) puerperal women with primary education or less, $437(60.5 \%)$ patients with secondary education and $98(13.6 \%)$ patients with higher education or more. Maternal characteristics differed in terms of age, previous pregnancies, normal deliveries, previous cesarean sections, age of 1st pregnancy, number of prenatal visits, income, number of people living in the household, and tobacco use. In the newborn profile, there was a difference only regarding cesarean sections. After calculating the odds ratio, it was seen that patients with primary education or less had a lower propensity to perform a cesarean section $(0.51195 \% \mathrm{Cl} 0.290-0.900)$, as well as patients with secondary education $(0.53095 \% \mathrm{Cl} 0.322-0.873)$, both compared to the population with higher education or more. In the other outcomes, no significance was observed. Conclusion: Primary and secondary education proved to be protective factors for cesarean section. No difference was found regarding prematurity, low birth weight, need for neonatal ICU and low 1st minute Apgar score.

Keywords: puerperae, schooling, Methodology

\section{INTRODUÇÃO}

Segundo o Instituto Brasileiro de Geografia e Estatística, no ano de 1997, $26 \%$ dos adolescentes frequentavam o ensino médio. Já no ano de $2007,48 \%$ dos adolescentes encontravam-se no ensino médio. Tal progressão, evidencia um aumento no grau da educação dos brasileiros. Não diferente desse aumento, as mulheres, antigamente, não presentes no ensino médio, mostraram-se mais 
frequentemente. O aumento também se fez presente nas universidades. A Síntese revelou que, nesses dez anos, subiu de 53,6\% para 57,1\% o percentual de mulheres entre os universitários. No ano de 2016, 23,5\% das mulheres brancas e 10,4\% das mulheres negras passaram a possuir ensino superior completo.

Diante dessa maior inserção da mulher no âmbito escolar e o aumento do seu grau nos últimos tempos, a ocorrência de gestação em mulheres acabou sofrendo alterações. As principais mudanças se fizeram no crescente número de mulheres que optam por postergar a maternidade, mulheres com idade maior ou igual a 35 anos, ocasionando no aumento do número de gestações em idade avançada nos últimos tempos. O desejo da mulher em investir na formação e na carreira profissional mostra-se como um dos fatores fundamentais que acabam por favorecer a gravidez tardia (ALVES e colab., 2017).

A ocorrência de gestação em mulheres com idade avançada está associada a eventos obstétricos adversos. Dentre os maiores riscos relacionados a gravidez em idade avançada e ao parto, pode-se ocorrer uma associação entre a maior frequência de doenças crônicas, tais quais hipertensão arterial e diabetes mellitus, e uma pior condição física da mulher. Abortos, anomalias cromossômicas, mortalidade materna, pré-eclâmpsia estão entre as principais complicações decorrentes da gestação tardia. Ainda, o grupo corresponde ao maior número de partos por via alta. Fatores tais quais solicitação materna, cirurgias prévias, placenta prévia, aumento de parto prematuro por complicações como pré-eclâmpsia contribuem para elevadas taxas de cesariana. Assim, a idade materna elevada torna-se um motivo de preocupação em relação a gestação e sua evolução resultando em um desafio a prática obstétrica. (LEITE e MONTEIRO, 2012)

Em relação às pacientes com menor escolaridade, a maioria dos estudos aponta que a baixa escolaridade materna está associada com a não realização do pré-natal acarretando em uma maior complicação de desfechos em decorrência da diminuição de consultas. A baixa escolaridade contribui para a não procura por atendimento e para com a realização de menor número de consultas. Já a maior escolaridade contribui para a realização de pré-natal. Analisando a menor escolaridade, mães com menos de 8 anos de escolaridade possuem uma chance de 1,5x maior chance de terem recém-nascidos com baixo peso. 
(QUADRADO e ROSA, 2014)

Estes fatores podem estar relacionados a um baixo padrão socioeconômico, menor ganho de peso na gestação e início mais tardio do prénatal. O número de consultas no pré-natal também se faz evidente já que mais com maior instrução possuem duas vezes mais chance de efetuarem mais de seis consultas no pré-natal. Ainda mães com menor escolaridade tendem a ter um maior número de filhos diminuindo o intervalo intergenésico predispondo a criança a um maior risco. (HAIDAR e colab., 2001)

A escolaridade mostra-se como um fator diferencial para com os desfechos maternos fetais adversos. Os diferentes níveis de escolaridade tendem a contribuir para o desenvolvimento de diversos fatores de riscos. Não só a mudança no qual as mulheres tendem a retardar a gestação visando investir na carreira profissional como também mulheres com baixa escolaridade optando por um pré-natal tardio. Ambos os grupos se firmam com um desafio a prática obstétrica. O objetivo desse artigo é comparar os diferentes níveis de escolaridade com os desfechos adversos perinatais em pacientes de baixo risco.

\section{METODOLOGIA}

Trata-se de um estudo corte transversal. Foi realizada a aplicação de uma entrevista que contemplou aspectos socioeconômicos e antecedentes obstétricos, além da análise de prontuário eletrônico a fim de avaliar o perfil do recém-nascido e dos desfechos adversos perinatais. Foi uma amostra estratificada de puérperas, correspondente a $10 \%$ da população atendida na Maternidade Darcy Vargas (MDV).

A amostra foi selecionada aleatoriamente através do programa "Randomized Research" através da indicação de 10 dias por mês, realizada no período de março de 2018 a fevereiro de 2019, no qual os pesquisadores deveriam aplicar as entrevistas a todas as puérperas que tiveram seus partos na Maternidade Darcy Vargas. A população foi dividida em 2 grupos, pacientes que fumaram durante a gestação e pacientes que não fumaram durante a gravidez.

A coleta de dados teve início após a autorização do Comitê de Ética em Pesquisa. O projeto foi aprovado sob o número CAAE 82477318.1.0000.5363 pelo Comitê de Ética em Pesquisa (CEP) do Hospital Regional Hans Dieter Schmidt, 
Joinville, SC, Brasil. O estudo seguiu os critérios definidos pela Resolução 466/2012 cada puérpera assinou o Termo de Consentimento Livre e Esclarecido presencialmente.

Os critérios de inclusão foram: puérperas com mais de 18 anos, com gestação única que realizaram o pré-natal em Unidades de Saúde do Sistema Único de Saúde da cidade de Joinville - SC que falavam português, cujo parto tenha ocorrido na Maternidade Darcy Vargas (MDV). O critério de exclusão de pacientes foi: puérperas que se recusaram a participar da pesquisa após o início do questionário.

Analisou-se os seguintes dados da população a fim de compreender seu perfil: características socioeconômicas da puérpera (idade, raça, escolaridade, renda, profissão remunerada, estado civil), uso de substâncias (álcool, e drogas) durante a gravidez, antecedentes obstétricos, complicações na gestação (DHEG, DMG e ITU), planejamento, perfil do pré-natal (número de consultas, IG da primeira consulta), características do recém-nascidos e desfechos adversos perinatais (prematuridade, internação em UTI neonatal e baixo peso ao nascer) até 48 horas após o parto. Todos os dados foram obtidos através de entrevista com escuta qualificada, consulta ao Prontuário Eletrônico.

Concomitantemente à coleta foi realizada a digitalização dos dados em um banco eletrônico com dupla entrada, para verificação de concordância e possíveis erros de digitação. Foi utilizado o software estatístico StatisticalPackage for the Social Sciences (SPSS), versão 21.0, para análise estatísticas dos dados. Todas as variáveis foram analisadas descritivamente, assim, as variáveis contínuas (numérica) foram estudadas por meio do cálculo de médias e desvios-padrão. Para as variáveis qualitativas calcularam-se frequências absolutas e relativas. Para a verificação da hipótese de igualdade entre as médias dos grupos, foi utilizado teste $\mathrm{T}$ de student, quando a distribuição foi normal, e o teste não paramétrico de Mann-Whitney, quando o teste de normalidade foi recusado. $O$ teste de normalidade utilizado foi o Kolmogorov-Smirnov. Para se provar a homogeneidade dos grupos em relação às proporções, utilizou-se o teste Quiquadrado ou o teste exato de Fisher para frequências abaixo de 5.

Modelos de regressão logística multinomial foram construídos de modo a analisar a influência da escolaridade sobre os desfechos estudados (internação 
em UTI neonatal, APGAR baixo de 1ำ minuto, prematuridade, baixo peso ao nascer e realização de cesariana). Fator de confusão utilizado: tabaco. Desse modo, estimou-se a relevância do efeito das variáveis pelo cálculo da razão de chances (OddsRatio - OR) ajustada conforme fatores de confusão, com seus respectivos intervalos de confiança de 95\% (IC95\%). Os valores foram considerados significativos quando $\mathrm{P}<0,05$.

\section{RESULTADOS}

Em nosso estudo, foram avaliadas 722 gestantes. Para melhor realizar a análise dos dados da pesquisa, as pacientes foram divididas em três grupos gestantes somente com o ensino primário $(n=187-25,90 \%)$, gestantes com 0 ensino secundário $(n=437-60,52 \%)$ e gestantes com ensino superior $(n=98-$ 13,57\%). O grupo analisado demonstra maior prevalência de pacientes com 0 ensino secundário tendo como objetivo comparar os graus de escolaridade e analisar seus desfechos perinatais.

Foram significativos $(p<0,05)$ Idade, gestações anteriores, partos normais, cesarianas prévias, idade da última gestação, número de consultas, profissão remunerada, número de pessoas na casa, tabacco.

Analisando as características dos recém-nascidos relacionados com ao grau de escolaridade materna, a indicação materna por cesariana mostrou-se a mais divergente. De acordo com os resultados obtidos, quanto maior a escolaridade maior a escolha médica por cesarianas. Assim, o grupo de gestantes com ensino superior teve uma necessidade por cesariana quando comparada com os grupos do ensino primário e ensino secundário. Os restantes das características tais quais Apgar, prematuridade, baixo peso a nascer e UTI neonatal não se mostraram importantes de acordo com o grau de escolaridade.

Após o cálculo de razão de chance, revelou-se que pacientes com ensino primário ou secundário tiverem menor propensão a realização de cesariana $(0,390$ IC95\% 0,224-0,678) quando comparados a puérperas com ensino superior. Também, segundo o estudo, pacientes com ensino secundário obtiverem menor propensão a realização de cesariana $(0,420$ IC95\% 0,258-0,683) em relação a puérperas com ensino superior. Ainda, utilizou-se o tabaco como um fator de confusão. O restante dos resultados não se mostrou significativos. 


\section{DISCUSSÃO}

Ao analisar os resultados obtidos durante a pesquisa, dentre os diversos fatores observados, a relação do número de cesáreas realizadas mostrou-se significativo quando comparado entre os três grupos de escolaridade. Mostrou-se uma relação direta entre o grau de escolaridade da paciente com a realização do parto por cesariana, onde puérperas que estudaram até o primário apresentavam uma menor prevalência em cesáreas, enquanto em pacientes com ensino superior esse índice demonstrou-se o mais alto.

A Organização mundial da Saúde (OMS) determinou que a taxa ideal de cesáreas seria em torno de $10 \%$ a $15 \%$ dos partos realizados; na prática, entretanto, os valores são mais elevados. De acordo com dados do Sistema de Informação sobre Nascidos Vivos (Sinasc), desde 1990, vem havendo um aumento no número de partos por cesárea, chegando a 55,7\% no ano de 2012. Visto que os números relacionados a partos pelo setor de Saúde Suplementar encontram-se estáveis desde 2005, o aumento de cesarianas mais recente pode ser associado aos partos realizados através do Sistema Único de Saúde.

Entre as pacientes da Maternidade Darcy Vargas que estudaram até o primário, 23,8\% passaram pelo procedimento de cesariana, contrastando com valores nacionais de 25,2\% para mulheres que nunca estudaram, 34,4\% com 1 a 3 anos de estudo e 40,1\% com 4 a 7 anos de escolaridade.

As pacientes com escolaridade até 0 secundário deste estudo apresentaram 31,95 de partos por cesariana, enquanto a média nacional para a mesma faixa de escolaridade fica acima, com $54,7 \%$. A relação se mantém com as puérperas que possuem ensino superior; No Brasil, a taxa de cesáreas é de $80,8 \%$, muito acima dos $50 \%$ obtidos neste estudo para pacientes com ensino superior (RATTNER e DE MOURA, 2016).

Quando comparada à taxa nacional, a prevalência de cesáreas ficou consideravelmente abaixo da média, segundo variáveis sociodemográficas de 2011. Isso indica que apesar de seguir o aumento padrão na incidência de cesáreas conforme aumenta a escolaridade, é possível constatar uma menor taxa de cesarianas desnecessárias quando comparada com a média do país.

Haidar (2001, p.1027) afirma que o impacto que o nível de escolaridade causa no tipo de parto pode decorrer tanto por opção da mãe quanto por influência 
médica, e por ser um procedimento que gera custos maiores, tende a ser mais frequente entre mulheres com maior escolaridade, que costumam se encontrar em melhores condições financeiras. Entretanto, as pacientes do presente estudo foram todas atendidas dentro do SUS, fazendo com que o fator financeiro não tivesse influência na taxa de cesáreas.

Ao avaliar as características maternas, foi observado que o número de gestações decai conforme o tempo de estudo aumenta. De acordo com Haidar (2001, p.1027), mães com menor escolaridade tendem a ter um número maior de filhos podendo ser decorrente da falta de informação ou menor acesso a medidas anticoncepcionais eficazes.

Além disso, a idade da primeira gestação também era maior conforme o grau de escolaridade aumentava. Entre as pacientes que estudaram até o primário apresentaram em média, uma idade menor na primeira gravidez (18,47 anos) enquanto que as que estudaram até o secundário tiveram sua gestação alguns anos mais tarde (20,81 anos) e entre as com ensino superior, a idade se mostrou, em média a mais elevada na primeira gestação (25,39 anos).

Tais informações somadas implicam que o acesso à educação favorece o uso de métodos contraceptivos, visto que entre as pacientes com menor escolaridade, não apenas o número de filhos é maior, como também a idade também tende a ser menor, apesar de a taxa de cesáreas ser menor entre gestantes desse grupo. Uma gravidez que aconteça muito cedo normalmente ocorre de forma não planejada, um acontecimento que costuma romper com a trajetória considerada ideal pela sociedade, como terminar a formação escolar, conseguir um emprego e alcançar sua autonomia financeira (RIBEIRO e GUALDA, 2011).

O nível de escolaridade, portanto, também se demonstrou impactante quando foi analisado se as pacientes possuíam emprego assalariado. A relação das que exercem uma profissão remunerada é diretamente proporcional ao nível de escolaridade. Os dados demonstraram que das que estudaram até o primário, apenas $36,4 \%$ exerciam profissão remunerada, enquanto $57,2 \%$ das que estudaram até o secundário e $78,6 \%$ das que tinham ensino superior exerciam alguma profissão. Dessa forma, fica evidente que uma maior escolaridade possibilita um acesso mais facilitado ao mercado de trabalho e a melhores 
condições de vida consequentemente.

Outro fator que se destacou na pesquisa foi incidência de tabagismo entre os grupos. Ficou evidente o impacto de um nível escolar maior exerce sobre o uso do cigarro. Dentre as pacientes, o tabagismo é maior naquelas do grupo com menor escolaridade, em que $22 \%$ disseram ser fumantes. Entre aquelas que estudaram até o secundário, 3,9\% disseram fazer uso de tabaco, enquanto nenhuma paciente dentre as que possuíam ensino superior disse ser fumante. Isso indica que o maior tempo de estudo reflete em um maior conhecimento a respeito dos danos causados pelo cigarro à saúde.

Entre os demais desfechos adversos perinatais analisados, a taxa de neonatos que foram encaminhados para a UTI neonatal não foi significativa. De acordo com um estudo realizado também em Joinville, observou-se que entre mulheres com menor escolaridade, a razão de chances era de 1,4 (IC95\% = 1,2 1,7) dos recém nascidos necessitaram de internação na UTI neonatal (MUCHA e colab., 2015), entretanto, os dados coletados para a faixa de escolaridade primária na presente pesquisa apontaram um valor de 1,05 (IC95\% = 0,180 - 6,115), com intervalo de confiança não adequado para estudo.

O índice de APGAR baixo no primeiro minuto não se fez significativo para o estudo. Apesar de ser um forte indicador de mortalidade infantil, não há como desconsiderar o impacto que fatores sociais podem causar em gestações de risco. Porém, como visto em estudos realizados em Cuiabá-MT (GAIVA e colab., 2016) e em Recife-PE (RIBEIRO e colab., 2009), dados como renda familiar ou grau de escolaridade não se encontram facilmente disponíveis nos sistemas de informações sobre nascidos vivos, o que inviabiliza a comparação desses indicadores para os fins do estudo.

O baixo peso ao nascer relacionado à escolaridade materna não foi significativo para ser avaliado, com razão de chances de 0,372 (IC95\% = 0,0592,339). De acordo com Carniel (2008, p.173-177), a escolaridade materna, sendo considerada um marcador de condição socioeconômica, foi significante para 0 baixo peso ao nascer, com razão de chances de 1,52 (IC95\% = 1,25 - 1,84) para até 7 anos de estudo e 1,36 (IC95\%=1,12 - 1,64) para mães com 8 a 11 de escolaridade.

A prematuridade dos neonatos também não foi significativa, com razão de 
chances de 4,347 (IC95\%=0,457-41,379). Em um estudo realizado no município de Parnamirim-RN (TEIXEIRA e colab., 2018), entretanto, foi verificado que o baixo nível de escolaridade e baixa renda estão relacionados à prematuridade; baixa escolaridade pode estar relacionada a maus hábitos de saúde e alimentação inadequada e obesidade devido à falta de informações (LINS e colab., 2013), além de também poder ser associada a outros fatores como pressão arterial elevada e uso de drogas durante a gestação, todos fatores de risco para nascimento prematuro.

\section{CONCLUSÃO}

Com base nos elementos acima apresentados, conclui-se que a necessidade por cesariana varia de acordo com o grau de escolaridade. Pacientes com o ensino superior tiveram uma maior escolha médica por cesariana quando comparado com pacientes com ensino secundário e ensino primário.

\section{Tabelas}

Tabela 1: características maternas relacionadas a escolaridade*

\begin{tabular}{|c|c|c|c|c|}
\hline & $\begin{array}{l}\text { Primário } \\
(n=187)\end{array}$ & $\begin{array}{l}\text { Secundário } \\
(n=437)\end{array}$ & $\begin{array}{l}\text { Superior } \\
(n=98)\end{array}$ & $\boldsymbol{P}$ \\
\hline Idade & $25,82(6,21)$ & $25,64(5,29)$ & $29,54(5,18)$ & $0,000 * *$ \\
\hline $\begin{array}{l}\text { Gestações } \\
\text { anteriores }\end{array}$ & $2,74(2,25)$ & $2,01(1,13)$ & $1,82(0,91)$ & $0,000 * *$ \\
\hline $\begin{array}{l}\text { Partos } \\
\text { normais }\end{array}$ & $1,81(1,55)$ & $1,30(1,04)$ & $0,87(0,90)$ & $0,000 * *$ \\
\hline $\begin{array}{l}\text { Cesarianas } \\
\text { previas }\end{array}$ & $0,70(1,05)$ & $0,51(0,82)$ & $0,73(0,87)$ & $0,016 * *$ \\
\hline Abortos & $0,22(0,59)$ & $0,20(0,49)$ & $0,21(0,43)$ & $0,867 * *$ \\
\hline $\begin{array}{l}\text { Idade 1a } \\
\text { gestação }\end{array}$ & $18,47(3,97)$ & $20,81(3,94)$ & $25,39(5,12)$ & $0,000 * *$ \\
\hline Planejamento & $62(33,2)$ & $169(38,7)$ & $35(35,7)$ & $0,412 * *$ \\
\hline IG 1a consulta & $10,01(5,71)$ & $8,55(4,17)$ & $9,02(4,50)$ & $0,195 * *$ \\
\hline $\begin{array}{l}\text { Número de } \\
\text { consultas }\end{array}$ & $7,72(2,19)$ & $8,44(2,18)$ & $8,67(2,22)$ & $0,000 * *$ \\
\hline \multicolumn{5}{|l|}{ Raças } \\
\hline Branca & $113(60,4)$ & $285(65,2)$ & $61(62,2)$ & $0,501 * *$ \\
\hline Negra & $45(24,1)$ & $106(24,3)$ & $22(22,4)$ & $0,930 * *$ \\
\hline Parda & $24(12,8)$ & $32(7,3)$ & $11(11,2)$ & $0,073 * *$ \\
\hline Outras & $5(2,7)$ & $14(3,2)$ & $4(4,1)$ & $0,813 * * *$ \\
\hline $\begin{array}{l}\text { Profissão } \\
\text { remunerada }\end{array}$ & $68(36,4)$ & $250(57,2)$ & $77(78,6)$ & $0,000 * *$ \\
\hline $\begin{array}{l}\text { Número de } \\
\text { pessoas na casa }\end{array}$ & $4,36(1,31)$ & $3,97(1,70)$ & $3,58(1,00)$ & $0,000 * *$ \\
\hline
\end{tabular}




$\begin{array}{lllll}\text { Tabaco } & 21(11,2) & 17(3,9) & 0(0,0) & 0,000^{* * *} \\ \text { Álcool } & 7(3,7) & 27(6,2) & 4(4,1) & 0,392^{* * *} \\ \text { Drogas } & 1(0,5) & 2(0,5) & 0(0,0) & 0,782^{* * *} \\ \text { DHEG } & 21(11,2) & 42(9,6) & 10(10,2) & 0,827^{* *} \\ \text { DMG } & 8(4,3) & 17(3,9) & 5(5,1) & 0,859^{* *} \\ \text { ITU } & 62(33,2) & 157(60,6) & 40(40,8) & 0,440 * *\end{array}$

*Média e desvio-padrão, números absolutos e percentagens; ${ }^{* *}$ Teste Qui-quadrado. ${ }^{* * *}$ Teste Exato de Fisher; IG - Idade Gestacional; DMG - Diabetes Mellitus Gestacional; DHEG - Doença Hipertensiva Especifica da Gestação; ITU- Infecção do Trato Urinário.

Tabela 2: características do recém-nascido relacionadas a escolaridade*

\begin{tabular}{|c|c|c|c|c|}
\hline & $\begin{array}{l}\text { Primário } \\
\text { ( } n=187)\end{array}$ & $\begin{array}{l}\text { Secundário } \\
(n=437)\end{array}$ & $\begin{array}{l}\text { Superior } \\
(n=98)\end{array}$ & $P$ \\
\hline Capurro & $39,02(1,64)$ & $38,94(1,70)$ & $39,12(1,32)$ & $0,162 * *$ \\
\hline Peso & $\begin{array}{l}3.304,59 \\
(453,50)\end{array}$ & $\begin{array}{l}3.355,56 \\
(522,34)\end{array}$ & $\begin{array}{l}3.341,58 \\
(410,30)\end{array}$ & $0,378 * *$ \\
\hline $\begin{array}{l}\text { APGAR de } 19 \\
\text { minuto }\end{array}$ & $7,84(1,04)$ & $7,73(1,10)$ & $7,77(0,94)$ & $0,657^{* *}$ \\
\hline $\begin{array}{l}\text { APGAR de 5o } \\
\text { minuto }\end{array}$ & $8,99(0,35)$ & $8,84(0,86)$ & $8,86(0,56)$ & $0,387^{* *}$ \\
\hline Cesariana & $53(23,8)$ & $126(31,9)$ & $44(50,0)$ & $0,003 * *$ \\
\hline Prematuridade & $9(4,8)$ & $23(5,2)$ & $4(4,1)$ & $0,882 * * *$ \\
\hline $\begin{array}{l}\text { Baixo peso ao } \\
\text { nascer }\end{array}$ & $10(5,3)$ & $20(4,6)$ & $3(3,1)$ & $0,680 * * *$ \\
\hline $\begin{array}{l}\text { APGAR baixo de } \\
19 \text { minuto }\end{array}$ & $13(7,0)$ & $25(5,7)$ & $9(9,2)$ & $0,436^{* *}$ \\
\hline $\begin{array}{l}\text { APGAR baixo de } \\
\text { 50 minuto }\end{array}$ & $1(0,5)$ & $1(0,2)$ & $0(0,0)$ & $0,684 * * *$ \\
\hline UTI neonatal & $6(3,2)$ & $17(3,9)$ & $3(3,1)$ & $0,874 * * *$ \\
\hline
\end{tabular}

*Média e desvio-padrão, números absolutos e percentagens; ${ }^{* *}$ Teste Qui-quadrado. ${ }^{* * *}$ Teste Exato de Fisher.

Tabela 3: razão de chance de desfechos adversos perinatais de acordo com a escolaridade

\begin{tabular}{|c|c|c|c|}
\hline & $P$ & RC & IC $95 \%$ \\
\hline \multicolumn{4}{|l|}{ PRIMÁRIO } \\
\hline UTI neonatal & 0,726 & 1,381 & $0,226-6,417$ \\
\hline $\begin{array}{l}\text { APGAR baixo de } \\
10 \text { minuto }\end{array}$ & 0,465 & 0,681 & $0,243-1,910$ \\
\hline $\begin{array}{l}\text { Baixo peso ao } \\
\text { nascer }\end{array}$ & 0,477 & 0,493 & $0,070-3,469$ \\
\hline Prematuridade & 0,318 & 3,362 & $0,311-35,406$ \\
\hline Cesariana & 0,020 & 0,511 & $0,290-0,900$ \\
\hline \multicolumn{4}{|l|}{ SECUNDÁRIO } \\
\hline UTI neonatal & 0,489 & 1,768 & $0,352-8,875$ \\
\hline $\begin{array}{l}\text { APGAR baixo de } \\
10 \text { minuto }\end{array}$ & 0,228 & 0,585 & $0,223-1,430$ \\
\hline $\begin{array}{l}\text { Baixo peso ao } \\
\text { nascer }\end{array}$ & 0,292 & 0,372 & $0,059-2,339$ \\
\hline Prematuridade & 0,201 & 4,347 & $0,457-41,379$ \\
\hline Cesariana & 0,013 & 0,530 & $0,322-0,873$ \\
\hline
\end{tabular}

Fator de confusão: tabaco e idade matern 


\section{REFERÊNCIAS}

ALVES, Nayara Cristina de Carvalho e colab. Complicações na gestação em mulheres com idade maior ou igual a 35 anos. Revista gaúcha de enfermagem, 2017.

HAIDAR, Fátima Hussein e OLIVEIRA, Urânia Fernandes e NASCIMENTO, Luiz Fernando Costa. Escolaridade materna: correlação com os indicadores obstétricos. Cadernos de Saúde Pública, 2001.

LEITE, Denise e MONTEIRO, Maia. Complicações maternas em gestantes. Femina, 2012.

QUADRADO, Cristiane e ROSA, Da. Fatores associados à não realização de prénatal em município de grande porte Factors associated with lack of prenatal care in a large municipality. Rev Saúde Pública, 2014.

World Health Organization. WHO Statement on Caesarean Section Rates. Human Reproduction Programme, Genebra, 2015.

RIBEIRO, Patricia Mônica e GUALDA, Dulce Maria Rosa. Gestação na adolescência: a construção do processo Saúde-Resiliência. Escola Anna Nery, v. 15, n. 2, p. 361-371, Jun 2011.

RATTNER, Daphne e DE MOURA, Erly Catarina. Nascimentos no Brasil: Associação do tipo de parto com variáveis temporais e sociodemográficas. Revista Brasileira de Saúde Materno Infantil, v. 16, n. 1, p. 39-47, 2016.

GAIVA, Maria Aparecida Munhoz e FUJIMORI, Elizabeth e SATO, Ana Paula Sayuri. MATERNAL AND CHILD RISK FACTORS ASSOCIATED WITH NEONATAL MORTALITY. v. 25, n. 4, p. 2290015, 2016. Disponível em: <http://dx.doi.org/10.1590/0104-07072016002290015>. Acesso em: 31 jan 2020.

LINS, Ana Paula Machado e colab. Alimentação saudável, escolaridade e excesso de peso entre mulheres de baixa renda. Ciencia e Saude Coletiva, v. 18, n. 2, p. 357-366, Fev 2013.

MUCHA, Fátima e colab. The frequency and characteristics of mothers and newborns associated with the admission of neonates to ICU in the municipality of Joinville, Santa Catarina-2012. Rev. Bras. Saúde Matern. Infant, v. 15, n. 2, p. 201208, 2015. Disponível em: <http://dx.doi.org/10.1590/S151938292015000200006 >. Acesso em: 13 jan 2020.

RIBEIRO, Adolfo Monteiro e colab. Fatores de risco para mortalidade neonatal em crianças com baixo peso ao nascer. Rev Saúde Pública. [S.I: s.n.], 2009. Disponível em: <http://www.monitorimi.cict.fi>. Acesso em: 31 jan 2020.

TEIXEIRA, Gracimary Alves e colab. Impact of maternal profile on birth outcomes. Cogitare Enfermagem, v. 23, n. 1, 2018 